\title{
Acervo de Formicidae (Insecta, Hymenoptera) da Coleção Entomológica Adolph Hempel, Instituto Biológico, São Paulo
}

\section{Collection of Formicidae (Insecta, Hymenoptera) in the Entomological Collection Adolph Hempel, Instituto Biológico, São Paulo}

\author{
Amanda Aparecida de Oliveiral, "I (1) | Kayhê Paiva Alves Cury Franco"II (D) | \\ Ingred Nobrega Teixeira"II (D) Wagner Yamakawa ${ }^{\text {IV }}$ (D) Sergio Ide III (D) \\ Instituto Biológico. Laboratório de Pragas Urbanas. São Paulo, São Paulo, Brasil \\ "Universidade Estadual Paulista. Programa de Pós-Graduação em Biologia Celular e Molecular. Rio Claro, São Paulo, Brasil \\ I'Instituto Biológico. Laboratório de Entomologia Geral. São Paulo, São Paulo, Brasil \\ "Projeto "Águas da Mantiqueira". Santo Antônio do Pinhal, São Paulo, Brasil
}

\begin{abstract}
Resumo: A preservação de informações a respeito de biodiversidade é muito importante para inúmeros campos da ciência. As coleções biológicas são responsáveis por guardar essas informações. Dentro da Mirmecologia, campo responsável pelos estudos das formigas, daremos destaque, neste texto, às informações históricas da Coleção Entomológica Adolph Hempel, Instituto Biológico de São Paulo, com especial referência ao acervo de formigas. A coleção de Formicidae contém 15.700 espécimes, identificados em 357 espécies, distribuídas entre 61 gêneros, pertencentes a dez subfamílias, com predomínio de Myrmicinae, Ponerinae, Formicinae e Dorylinae, que constituem 78\% do acervo. Em relação ao material-tipo, o acervo contém 16 espécimes, distribuídos entre noves espécies.
\end{abstract}

Palavras-chave: Formigas. Tomás Borgmeier. Walter Kempf.

Abstract: Preserving information about biodiversity is crucial for many fields of science, and biological collections are responsible for keeping this information. Within myrmecology, the scientific study of ants, we highlight in this article historical information from the Entomological Collection Adolph Hempel, Instituto Biológico of Sao Paulo, with special reference to the ant collection. The Formicidae collection contains 15,700 specimens, identified in 357 species, which are distributed among 61 genera belonging to 10 subfamilies. There is a predominance of Myrmicinae, Ponerinae, Formicinae, and Dorylinae, which together constitute 78\% of the collection. Type material deposited in the repository is represented by 16 specimens, distributed in nine species.

Keywords: Ants. Tomás Borgmeier. Walter Kempf.

OLIVEIRA, A. A., K. P. A. C. FRANCO, I. N. TEIXEIRA, W. YAMAKAWA \& S. IDE, 2020. Acervo de Formicidae (Insecta, Hymenoptera) da Coleção Entomológica Adolph Hempel, Instituto Biológico, São Paulo. Boletim do Museu Paraense Emílio Goeldi. Ciências Naturais 15(1): 307-316. DOI: http://doi.org/10.46357/bcnaturais.v15i1.259.

Autora para correspondência: Amanda Aparecida de Oliveira. Centro de Estudos de Insetos Sociais. Avenida 24A, 1515, Bela Vista. Rio Claro, SP, Brasil. CEP 13506-900 (oliveira.amandah@gmail.com).

Recebido em 15/01/2020

Aprovado em 10/04/2020

Responsabilidade editorial: Lívia Pires do Prado
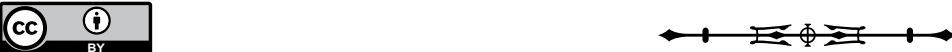


\section{ORIGENS DO INSTITUTO BIOLÓGICO}

Em meados da década de 1920, o estado de São Paulo era considerado o maior produtor de café (Coffea spp.) (Rubiaceae) do país. Entretanto, nessa época, inúmeras áreas de plantio da cultura estavam arruinadas pela broca-do-café, Hypothenemus hampei (Ferrari, 1867), um besouro que ataca a coroa do grão, perfurando a semente para se alimentar. Em decorrência da infestação da broca, houve grande expansão de pesquisas para o combate de pragas agrícolas. Neste contexto, no ano de 1924, o governo do estado de São Paulo instituiu a Comissão de Estudo e Debelação da Praga Cafeeira, convidando para fazer parte de seu corpo o então diretor do Museu Nacional do Rio de Janeiro, Arthur Neiva. $O$ objetivo era encontrar uma forma de controle para a praga que devastava a principal fonte de renda do estado paulista. Como a missão foi exitosa, uma proposta para a criação de um instituto para promoção de "pesquisas em benefício da lavoura e da pecuária" foi feita por Neiva (Kempf, 1976). Esta proposta foi o projeto inicial do Instituto Biológico (IB), fundado dia 26 de dezembro no ano de 1927 (Kempf, 1976; Rebouças, 2009; Rebouças \& Batista Filho, 2017).

No final da mesma década, Adolph Hempel, então assistente-chefe da sessão de Entomologia e Parasitologia Animal do IB, foi responsável por um dos primeiros controles biológicos de pragas na agricultura do Brasil, trazendo, em 1929, a vespinha-da-uganda, Prorops nasuta (Waterson, 1923), para controlar a praga do café (Rebouças, 2006).

Contudo, fundou-se o Instituto Biológico e assim nasceu a coleção, que inicialmente estava destinada a abrigar o material das pesquisas relacionadas à área agrícola. As coleções biológicas são os acervos que compõem bancos de materiais (espécimes) vivos ou preservados, associados aos dados biológicos e geográficos. As informações contidas nesses acervos podem ser utilizadas como ferramentas imprescindíveis para o trabalho dos taxonomistas e como apoio indispensável para muitas outras áreas do conhecimento (Peixoto et al., 2006).
As três principais coleções zoológicas brasileiras são do Museu de Zoologia da Universidade de São Paulo (MZSP), o Museu Nacional do Rio de Janeiro (MNRJ) e o Museu Paraense Emílio Goeldi (MPEG), por serem centenárias e abrigarem grande quantidade de material (De Vivo et al., 2014). Entretanto, outras instituições destacaram-se para a área de entomologia, como a Universidade Federal do Paraná (UFPR) e o Instituto Nacional de Pesquisas da Amazônia (INPA).

Neste artigo, tratamos da Coleção Entomológica Adolph Hempel (IBSP-IB) (Figuras 1A e 1B). A coleção foi estabelecida no final da década de 1920 por José Pinto da Fonseca (Ide et al., 2005), mas somente em 31 de julho de 1994 que recebeu este nome, em homenagem ao eminente entomólogo e pesquisador do Instituto Biológico, Adolph Hempel. Alguns aspectos que tornam o acervo relevante são: (i) a quantidade de espécimes - pois é considerada a segunda maior coleção do estado de São Paulo em número de espécimes; (ii) o acervo de cochonilhas (Hemiptera, Coccoidea) mais importante do Brasil, considerado referência mundial para o estudo de Coccoidea, incluindo grande quantidade de tipos descritos por Hempel e Fonseca, entre outros pesquisadores; (iii) é repositório de diversas espécies-tipo de diferentes ordens de Insecta; e (iv) importância histórica pelos exemplares-testemunho de projetos desenvolvidos pelos pesquisadores do Instituto Biológico desde a sua fundação (Cytrynowicz et al., 2013).

A coleção entomológica do IBSP-IB abriga espécies e material-tipo de diversos projetos de importância econômica que fazem parte da história do Instituto Biológico, sendo eles: biologia, comportamento, sistemática e controle de pragas agrícolas, como broca-do-café, moscas-das-frutas e formigas cortadeiras, o que torna esta coleção uma das maiores fontes de informação sobre insetos com importância econômica do Brasil (Costa et al., 2000).

Sob curadoria do Dr. Sergio Ide, o acervo está abrigado em salas de $81 \mathrm{~m}^{2}$ e $40 \mathrm{~m}^{2}$, no Laboratório de Entomologia Geral, no $6^{\circ}$ andar do Edifício Central do IB. Estima-se que o total de espécimes e lotes do material

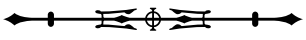




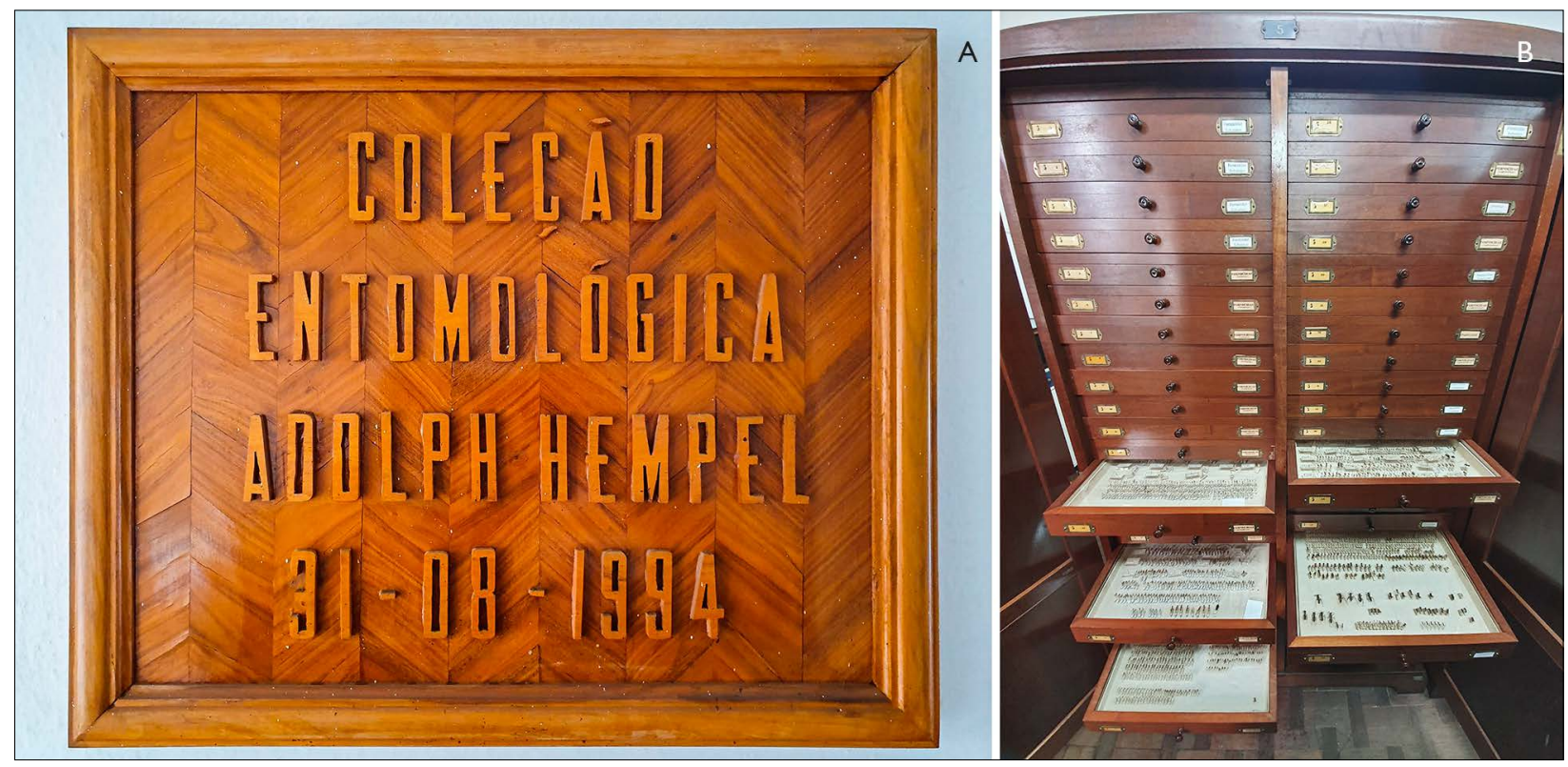

Figura 1. Coleção Entomológica Adolph Hempel (IBSP-IB): A) placa da Coleção; B) armário do acervo de Formicidae da Coleção. Fotos: I. N. Teixeira (2020).

preparado e incorporado à parte principal da coleção em via seca seja superior a 300.000 espécimes, referente a 15 ordens e a cerca de 350 famílias, sendo 250 espécimes e quatro famílias de Odonata; 250 espécimes e dez famílias de Orthoptera; 20 espécimes e uma família de Phasmatodea; 50 espécimes e quatro famílias de Blattodea; 4.000 lotes e dez famílias de Coccoidea (Hemiptera); 40.000 espécimes e 73 famílias de Heteroptera (Hemiptera); 151.000 espécimes e 60 famílias de Coleoptera; 10.000 espécimes e 20 famílias de Diptera; 15.000 espécimes e 36 famílias de Lepidoptera; 65.000 espécimes e 26 famílias de Hymenoptera; 15.000 espécimes distribuídas entre outras ordens (Ide et al., 2005). Em setembro de 2015, a coleção entomológica do Instituto Agronômico de Campinas (ex-coleção IAC), São Paulo, foi incorporada ao IBSP-IB. O acervo desta coleção inclui mais de 25.000 espécimes das principais ordens de Insecta.

\section{ACERVO DE FORMICIDAE: HISTÓRICO E COMPOSIÇÃO}

Um dos nomes considerados mais importantes na formação da coleção do acervo de Formicidae é o de
Tomás Borgmeier, cujo vínculo data desde antes de sua fundação. Além dos trabalhos realizados por Borgmeier, metade de seu acervo particular de formigas foi adquirido pelo IB (Horn et al., 1990).

Nesta época, Tomás Borgmeier foi convidado por Artur Neiva para integrar a equipe de pesquisadores da instituição (Figura 2), que tinha como foco pesquisas com espécies de importância agrícola, especialmente saúvas (formigas-cortadeiras) (Kempf, 1976). Suas contribuições foram diversificadas e abrangeram desde observações sobre a história natural e descrição de espécies (Borgmeier, 1939) até discussões taxonômicas acaloradas sobre a validade de espécies descritas (Borgmeier, 1931, 1932; Costa Lima, 1931). Desde o início dos trabalhos no IB, Borgmeier contou com laboratórios para desenvolver sua pesquisa, com auxiliares e equipamentos para elaborar os estudos que resultaram em diversos artigos publicados em revistas da instituição; foi também mentor dos entomólogos John Lane e Mário Autuori, este último autor de diversos estudos principalmente sobre biologia e controle de formigas-cortadeiras (Rebouças \& Batista Filho, 2017).

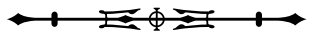


Borgmeier era citado como um "homem de produção, de trabalho insano e persistente" (Kempf, 1976). Toda a sua dedicação foi primordial para geração e ampliação do conhecimento sobre Formicidae que se tinha à época, contribuindo de forma decisiva para o incremento e a consolidação do acervo (Kempf, 1976). Dessa maneira, é impossível dissociar seu nome da história da coleção de formigas do IB.

O núcleo inicial do acervo de Formicidae do IBSP-IB foi composto por parte da coleção particular de Borgmeier. Como ele necessitava de fundos para a criação da Revista de Entomologia, a transferência foi efetuada através da compra de metade da coleção pelo IB. A outra metade da coleção foi incorporada à coleção de Walter Kempf, a qual, posteriormente, foi transferida para o Museu de Zoologia da Universidade de São Paulo (Horn et al., 1990).

Atualmente, o acervo é composto por cerca de 15.700 espécimes, pertencentes a 357 espécies, 61 gêneros e dez subfamílias, com predomínio de Myrmicinae, Ponerinae, Formicidae e Dorylinae, que constituem 78\% do acervo (Tabelas 1 e 2). Os exemplares estão fixados em alfinetes entomológicos, distribuídos em 52 gavetas (Figura 3A).

Tabela 1. Número de gêneros e espécies de Formicidae (Insecta, Hymenoptera) por subfamília da Coleção Entomológica Adolph Hempel, Instituto Biológico, São Paulo, e ex-coleção do Instituto Agronômico de Campinas.

\begin{tabular}{c|c|c}
\hline Subfamília & Número de gêneros & Número de espécies \\
\hline Amblyoponinae & 1 & 1 \\
\hline Dolichoderinae & 6 & 36 \\
\hline Dorylinae & 6 & 39 \\
\hline Ectatomminae & 2 & 14 \\
\hline Formicinae & 7 & 76 \\
\hline Heteroponerinae & 2 & 3 \\
\hline Myrmicinae & 25 & 139 \\
\hline Paraponerinae & 1 & 1 \\
\hline Ponerinae & 10 & 36 \\
\hline Pseudomyrmecinae & 1 & 12 \\
\hline Total & 61 & 357
\end{tabular}

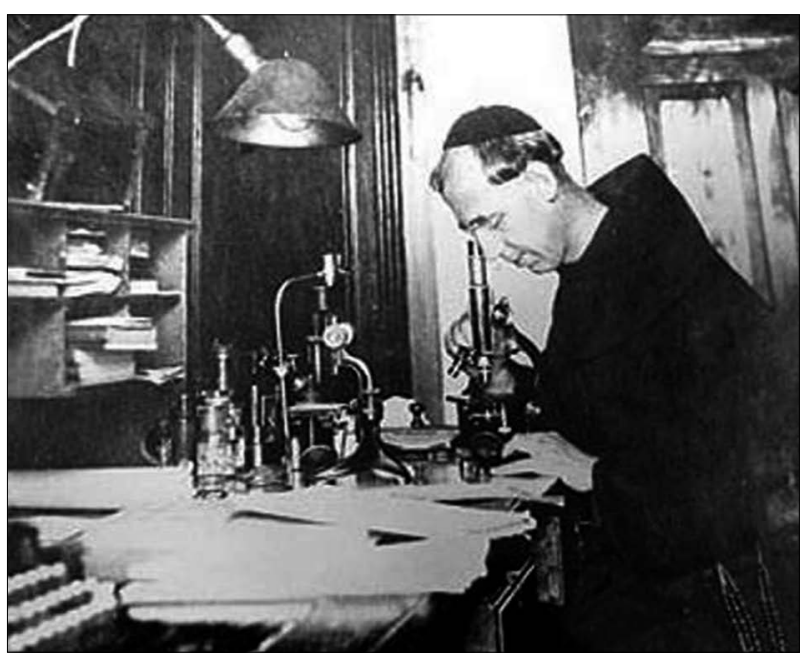

Figura 2. Frei Tomás Borgmeier trabalhando no Instituto Biológico. Fonte: Garmus (2011).

Tabela 2. Lista de gênero e número de espécies depositados na Coleção IBSP-SP.

(Continua)

\begin{tabular}{|c|c|c|}
\hline Subfamília & Gêneros & Número de espécies \\
\hline Amblyoponinae & Fulakora & 1 \\
\hline \multirow{6}{*}{ Dolichoderinae } & Azteca & 9 \\
\hline & Dolichoderus & 13 \\
\hline & Dorymyrmex & 4 \\
\hline & Forelius & 3 \\
\hline & Linepithema & 6 \\
\hline & Tapinoma & 1 \\
\hline \multirow{6}{*}{ Dorylinae } & Acanthostichus & 4 \\
\hline & Eciton & 11 \\
\hline & Cylindromyrmex & 1 \\
\hline & Labidus & 4 \\
\hline & Neivamyrmex & 17 \\
\hline & Nomamyrmex & 2 \\
\hline \multirow{2}{*}{ Ectatomminae } & Ectatomma & 8 \\
\hline & Gnampotogenys & 6 \\
\hline \multirow{7}{*}{ Formicinae } & Acropyga & 1 \\
\hline & Brachymyrmex & 6 \\
\hline & Camponotus & 54 \\
\hline & Gigantiops & 1 \\
\hline & Myrmelachista & 9 \\
\hline & Nylanderia & 3 \\
\hline & Paratrechina & 2 \\
\hline
\end{tabular}

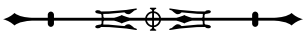


Tabela 2.

\begin{tabular}{|c|c|c|}
\hline Subfamília & Gêneros & Número de espécies \\
\hline \multirow{2}{*}{ Heteroponerinae } & Acanthoponera & 1 \\
\hline & Heteroponera & 2 \\
\hline \multirow{25}{*}{ Myrmicinae } & Acromyrmex & 17 \\
\hline & Adelomyrmex & 0 \\
\hline & Apterostigma & 1 \\
\hline & Atta & 7 \\
\hline & Cephalotes & 12 \\
\hline & Crematogaster & 18 \\
\hline & Cyphomyrmex & 4 \\
\hline & Daceton & 1 \\
\hline & Leptothorax & 0 \\
\hline & Megalomyrmex & 6 \\
\hline & Monomorium & 3 \\
\hline & Mycetarotes & 1 \\
\hline & Mycocepurus & 1 \\
\hline & Myrmicocrypta & 1 \\
\hline & Nesomyrmex & 2 \\
\hline & Ochetomyrmex & 0 \\
\hline & Pheidole & 35 \\
\hline & Pogonomyrmex & 1 \\
\hline & Procryptocerus & 5 \\
\hline & Sericomyrmex & 1 \\
\hline & Solenopsis & 15 \\
\hline & Strumigenys & 3 \\
\hline & Tetramorium & 2 \\
\hline & Tranopelta & 1 \\
\hline & Wasmannia & 2 \\
\hline Paraponerinae & Paraponera & 1 \\
\hline \multirow{10}{*}{ Ponerinae } & Anochetus & 2 \\
\hline & Centromyrmex & 0 \\
\hline & Dinoponera & 2 \\
\hline & Hypoponera & 10 \\
\hline & Leptogenys & 3 \\
\hline & Mayaponera & 1 \\
\hline & Neoponera & 9 \\
\hline & Odontomachus & 4 \\
\hline & Pachycondyla & 4 \\
\hline & Platythyrea & 1 \\
\hline Pseudomyrmecinae & Pseudomyrmex & 12 \\
\hline Total & 61 & 357 \\
\hline
\end{tabular}

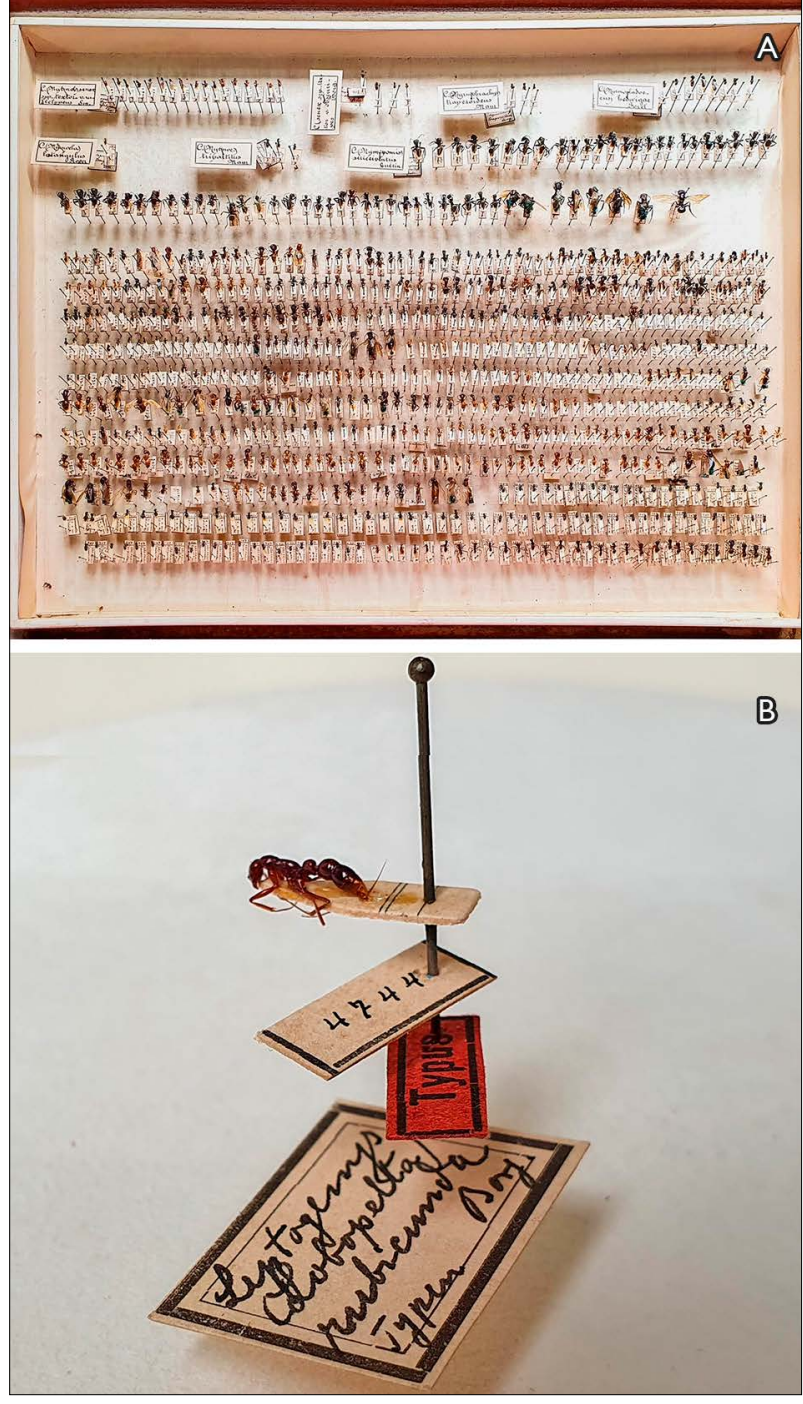

Figura 3. A) Gaveta do acervo de Formicidae da Coleção Entomológica Adolph Hempel em vista superior; B) espécimetipo de Leptogenys rubicunda Borgmeier, 1930. Fotos: I. N. Teixeira (2020).

material da ex-coleção IAC é composto de 47 espécimes, mantidos em duas gavetas entomológicas, incluindo espécimes das subfamílias Dolichoderinae, Dorylinae, Ectatomminae, Formicinae, Myrmicinae, Ponerinae e Pseudomyrmecinae. Todos os espécimes das gavetas foram contabilizados com auxílio de contador manual, e as informações quanto ao gênero e às espécies foram verificadas em AntWeb (2020). 


\section{ESPÉCIES-TIPO}

No acervo, estão depositados 16 espécimes-tipo pertencentes a nove espécies (Tabela 3, Figura 3B). O Anexo apresenta a transcrição dos rótulos dos espécimes-tipo. Foram encontrados exemplares rotulados como tipos, os quais, contudo, não foram confirmados, sendo eles: Pheidole rufipilis Forel, 1908; P. claviscapa Santschi, 1925; P. eidmanni Menozzi, 1926; P. gulelmimuelleri Forel, 1886; Camponotus senex r. textor Forel, 1899; Megalomyrmex iheringi Forel, 1911; e Dolichoderus (Hypoclinea) germainigarbei Forel, 1911. Apesar de esses exemplares estarem com rótulos de tipo, não existem informações sobre isso nos livros-tombo e nas publicações originais, necessitando de uma investigação detalhada no futuro.

\section{O ACERVO NOS DIAS DE HOJE}

Recentemente, o acervo da IBSP-IB tem sido enriquecido por material coletado pelo projeto "Águas da Mantiqueira", iniciado em abril de 2017 e financiado pela Fundação
Toyota do Brasil, São Paulo (Nicolau, 2019). O projeto visa conhecer e mensurar a diversidade de insetos (Arthropoda, Insecta), peixes (Osteichthyes), anfíbios (Amphibia), aves (Reptiliomorpha), mamíferos (Mammalia) (Chordata, Vertebrata) e flora da serra da Mantiqueira, com vistas à proposição de medidas de conservação e elaboração dos planos diretores dos municípios da região. As áreas de coleta foram delimitadas pelas bacias hidrográficas e, embora as coletas de insetos não sejam exclusivas para Formicidae, 92 espécimes da família, provenientes de 40 bacias hidrográficas de Santo Antônio do Pinhal, São Paulo, e Sapucaí-Mirim, Minas Gerais, já foram coletados. Este ano o projeto continuará no município de Sapucaí-Mirim, Minas Gerais, percorrendo as demais 21 bacias.

A equipe do Instituto Biológico que integra o projeto é composta por um pesquisador, um biólogo e um estagiário, que auxiliam na montagem, na identificação e no armazenamento do material coletado.

Tabela 3. Material-tipo de Formicidae (Insecta, Hymenoptera) depositado na Coleção Entomológica Adolph Hempel, Instituto Biológico, São Paulo. O status dos tipos foi transcrito de acordo com os dados de rótulo.

\begin{tabular}{|c|c|c|c|c|}
\hline Espécie & Nome válido & Status & $\begin{array}{l}\text { Número de } \\
\text { exemplares }\end{array}$ & Referências \\
\hline Azteca aesopus Forel, 1908 & $\begin{array}{l}\text { A. aesopus } \\
\text { Forel, } 1908\end{array}$ & Síntipo & 2 & Forel (1908) \\
\hline Camponotus scrobifer Borgmeier, 1928 & $\begin{array}{l}\text { C. coriolanus } \\
\text { Forel, } 1912\end{array}$ & Tipo & 1 & Borgmeier (1928) \\
\hline Camponotus subtruncatus Borgmeier, 1929 & $\begin{array}{l}\text { C. subtruncatus } \\
\text { Borgmeier, } 1929\end{array}$ & Tipo & 2 & Borgmeier (1929) \\
\hline Leptogenys (Lobopelta) hanseni Borgmeier, 1930 & $\begin{array}{l}\text { L. australis } \\
\text { Emery, } 1888\end{array}$ & Síntipo & 2 & Borgmeier (1930) \\
\hline Leptogenys (Lobopelta) rubicunda Borgmeier, 1930 & $\begin{array}{l}\text { L. crudelis } \\
\text { Smith, } 1858\end{array}$ & Tipo & 3 & Borgmeier (1930) \\
\hline $\begin{array}{c}\text { Megalomyrmex (Wheelerimyrmex) humilis } \\
\text { Borgmeier, } 1930\end{array}$ & $\begin{array}{l}\text { P. gertrudae } \\
\text { Forel, } 1886\end{array}$ & Holótipo & 1 & Borgmeier (1930) \\
\hline Megalomyrmex brasiliensis Borgmeier, 1930 & $\begin{array}{c}\text { M. silvestrii } \\
\text { Wheeler, } 1909\end{array}$ & Síntipo & 3 & Borgmeier (1930) \\
\hline Paracryptocerus fleddermanni Kempf, 1958 & $\begin{array}{c}\text { Cephalotes notatus } \\
\text { Mayr, } 1866\end{array}$ & Parátipo & 1 & Kempf (1958) \\
\hline Paracryptocerus frigidus Kempf, 1960 & $\begin{array}{l}\text { Cephalotes frigidus } \\
\text { (Kempf, 1960) }\end{array}$ & Holótipo & 1 & Kempf (1960) \\
\hline Total & & & 16 & \\
\hline
\end{tabular}

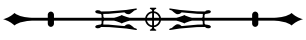




\section{NECESSIDADES E DESAFIOS}

Podemos ressaltar que, após 26 anos de sua oficial nomeação, uns dos principais desafios para a coleção Adolph Hempel (IBSP-SP) é a expansão para melhor acomodação do acervo e inclusão de novos exemplares. Para isso, é necessário modificar o sistema de armazenamento dos atuais armários de madeira para sistema de armários deslizantes, que proporcionarão grande ganho de espaço. Outro ponto importante refere-se à falta de pessoas trabalhando com a coleção entomológica, uma vez que a escassez de alunos e pesquisadores impede a realização de diversos trabalhos na coleção do Instituto Biológico, desde auxilio para a sua composição até a digitalização do acervo. Vale ressaltar que a digitalização da coleção está em andamento, contudo o processo ocorre de maneira lenta, em razão da falta de pessoal.

\section{AGRADECIMENTOS}

A. A. Oliveira agradece à Coordenação de Apoio ao Pessoal de Nível Superior(CAPES), pela concessão da bolsa de doutorado; K. P. A. C. Franco, I. N. Teixeira, W. Yamakawa e S. Ide agradecem à Fundação Toyota do Brasil, pelo apoio financeiro; à Márcia Maria Rebouças, pelo auxílio no levantamento de material histórico bibliográfico do Instituto Biológico.

\section{REFERÊNCIAS}

ANTWEB, 2020. AntWeb Version 8.22. California Academy of Science. Disponível em: https://www.antweb.org. Acesso em: 10 janeiro 2020.

BORGMEIER, T., 1928. Algumas formigas do Museo Paulista. Boletim Biológico (12): 55-70.

BORGMEIER, T., 1929. Zur Kenntnis der brasilianischen Ameisen. EOS. Revista Española de Entomología 5(2): 195-214.

BORGMEIER, T., 1930. Duas rainhas de Eciton e algumas outras formigas brasileiras. Archivos do Instituto Biologico de Defesa Agricola e Animal 3: 21-40, ests. 1-6.

BORGMEIER, T., 1931. Pequenas communicações: Acropyga pickeli Borgm. 1927 (Hym., Formicidae). Revista de Entomologia 1(1): 105-106.

BORGMEIER, T., 1932. Pequenas communicações: a proposito de Acropyga pickeli Borgm. (1927) (Hym. Formicidae). Revista de Entomologia 2(2): 238-239.
BORGMEIER, T., 1939. Nova contribuição para o conhecimento das formigas neotropicais (Hym. Formicidae). Revista de Entomologia 10(2): 403-428.

COSTA, C., S. IDE, G. H. ROSADO-NETO, M. H. M. GALILEO, C. R. V. FONSECA, R. M. VALENTE \& M. A. MONNÉ, 2000. Diagnóstico del conocimiento de las principales colecciones brasileñas de Coleoptera. In: F. MARTÍN-PIERA, J. J. MORRONE \& A. MELIC (Ed.): Hacia un Proyecto CYTED para el inventario y estimación de la diversidad entomológica en Iberoamérica: PriBES 2000: v. 1: 115-136. Sociedad Entomológica Aragonesa (SEA)/ Monografías Tercer Milenio, Zaragoza.

COSTA LIMA, A., 1931. A propósito da Acropyga pickeli Borgm., 1927 (Hymenoptera: Formicoidea). Boletim Biologico (17): 2-9.

CYTRYNOWICZ, R., M. M. REBOUÇAS \& S. D'AGOSTINI, 2013. Álbum histórico do Instituto Biológico: 86 anos de ciência em sanidade animal e vegetal. Editora Narrativa Um, São Paulo.

DE VIVO, M., L. F. SILVEIRA \& F. O. DO NASCIMENTO, 2014. Reflexões sobre coleções zoológicas, sua curadoria e a inserção dos Museus na estrutura universitária brasileira. Arquivos de Zoologia 45: 105-113. DOI: https://doi.org/10.11606/issn.2176-7793. v45iespp105-113.

FOREL, A., 1908. Ameisen aus Sao Paulo (Brasilien), Paraguay etc. gesammelt von Prof. Herm. v. Ihering, Dr. Lutz, Dr. Fiebrig, etc. Verhandlungender Kaiserlich-Königlichen ZoologischBotanischen Gesellschaftin Wien 58(8-9): 340-418.

GARMUS, L., 2011. Tómas Borgmeier o cientista. Revista Instituto Teológico Franciscano 3: 23-27. Disponível em: https://issuu.com/ provinciafranciscana/docs/121009234629-fafdd963efbe4c079d095 d72f2fc6243/23. Acesso em: 30 abril 2020.

HORN, W., I. KAHLE, G. FRIESE \& R. GAEDIKE, 1990. Collectiones entomologicae: Ein Kompendium über den Verbleib entomologischer Sammlungen der Welt bis 1960. Teil I: A bis K. Akademie der Landwirtschaftswissenschaften der Deutschen Demokratischen Republik, Berlin.

IDE, S., J. E. R. MARTINS, A. E. C. CAMPOS-FARINHA, S. L. IMENES \& W. YAMAKAWA, 2005. Coleção Entomológica Adolph Hempel, Instituto Biológico - São Paulo: história, importância e função. Páginas do Instituto Biológico 1(1): 1-40. Disponível em: http://www.biologico.sp.gov.br/uploads/docs/pag/v1_1/ide.htm. Acesso em: 19 novembro 2019.

KEMPF, W., 1958. New studies of the ant tribe Cephalotini (Hym. Formicidae). Studia Entomologica (n. s.) 1(1-2): 1-168. DOI: https:// doi.org/10.5281/zenodo.26000.

KEMPF, W., 1960. Miscellaneous studies on Neotropical ants (Hymenoptera, Formicidae). Studia Entomologica (n. s.) 3(1-4): 417-466. DOI: https://doi.org/10.5281/zenodo.26016. 
KEMPF, W., 1976. Frei Tomás Borgmeier (1892-1975). Vida Franciscana (50): 77-96. Disponível em: https://franciscanos.org. br/quemsomos/personagens/frei-tomas-borgmeier/. Acesso em: 19 novembro 2019.

NICOLAU, S. A. (Org.), 2019. Almanaque de Santo Antônio do Pinhal: qualidade de vida para os pinhalenses, transformando nossa cidade. Fundação Toyota do Brasil \& Fundação de Desenvolvimento da Pesquisa do Agronegócio, São Paulo.

PEIXOTO, A. L., M. R. D. V. BARBOSA, M. MENEZES \& L. C. MAIA, 2006. Diretrizes e estratégias para a modernização de coleções botânicas brasileiras com base na formação de taxonomistas e na consolidação de sistemas integrados de informação sobre biodiversidade. In: A. L. PEIXOTO, M. R. D. V. BARBOSA, M. MENEZES, L. C. MAIA, R. F. VAZOLELER, L. MARINONI \& D. A. L. CANHOS (Org.): Diretrizes e estratégias para a modernização de coleções biológicas brasileiras e a consolidação de sistemas integrados de informação sobre biodiversidade: 145-182. Centro de Gestão e Estudos Estratégicos/Ministério da Ciência e Tecnologia, Brasília.
REBOUÇAS, M. M., 2006. Pelo resgate da memória documental das ciências e da agricultura: o acervo do Instituto Biológico de São Paulo. História, Ciências, Saúde-Manguinhos 13(4): 995-1005. DOI: https://doi.org/10.1590/S0104-59702006000400011.

REBOUÇAS, M. M. (Org.), 2009. Arthur Neiva, o idealizador do Instituto Biológico. Instituto Biológico, São Paulo.

REBOUÇAS, M. M. \& A. BATISTA FILHO (Org.), 2017. Instituto Biológico: 90 anos inovando o presente. Editora Narrativa Um, São Paulo.

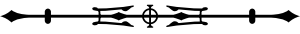


Anexo. Transcrição das informações dos rótulos dos espécimes-tipo de Formicidae (Insecta, Hymenoptera) da Coleção Entomológica Adolph Hempel, Instituto Biológico, São Paulo. Legendas: 01 = informações contidas nos rótulos; 02 = informações do livro de tombo. Nos rótulos, " [ ] " delimitam informações de cada rótulo e " | " delimita informações de linha dos rótulos. No livro de tombo, " [ ]" delimitam informações de cada registro, " || " delimitam informações de colunas e " | " delimita informações de cada linha. Quando o termo "Ibid." constava no registro, as informações correspondentes foram citadas entre parênteses.

(Continua)

Azteca aesopus Forel, 1908

01. [ 2155 ] [ Cotypus ] [ BRASIL, EsPírITO SAN | TO ] [aesopus | For. | Calypen ] [ Coleção Entomológica Adolph | Hempel - Instituto Biológico | IBSP-IB-0.001.875 ].

02. [ 2155 || Asteca aesopus For. | Esp. Santo. ].

Camponotus scrobifer Borgmeier, 1928

01. [ 3984 ] [ Typus ] [ BRASIL, São PAulo, | Guarujá, 1910 ] [ Campon. | Neocolobopsis | scrobifer | Borg. ] [C. Neocolobopsis | scrobifer Borg. | Typus | det Borgmeier] [Coleção Entomológica Adoph | Hempel - Instituto Biológico | IBSP-IB-0.001.878 ].

02. [3984 || Camponotus scroider Borgm. | G Guarujá. S. P. 1910. ].

Camponotus subtruncatus Borgmeier, 1929

01. [ 57 ] [ C. (Pseudocol.) | subtruncatus | Borg. ] [ BRASIL, RIO DE JANEI- | RO, Petrópolis. 1918 | Borgmeier col. ] [ Coleção Entomológica Adoph | Hempel - Instituto Biológico | IBSP-IB-0.001.877 ].

01. [ 57 ] [ BRASIL, RIO DE JANEI- | RO, Petrópolis. 1918 | Borgmeier col. ] [ Coleção Entomológica Adoph | Hempel - Instituto Biológico | IBSP-IB-0.001.884 ].

02. [57. || Camponotus subtruncatus Borgm. | Petrop. Borgm. XI.1918 ].

Leptogenys hanseni Borgmeier, 1930

01. [3616] [ 9 ] [ Typus] [BRASIL, Rıo Grande | do Sul, Pareci Novo, | X.1927, Hansen col.] [ Coleção Entomológica Adoph | Hempel - Instituto Biológico | IBSP-IB-0.001.178 ].

02. [ 3616 || Leptogenys hanseni Borg. | Ibid. (Hid. Pareci Novo X.27. Hansen) ].

Leptogenys rubicunda Borgmeier, 1930

01. [ 4744 ] [ Typus ] [ Leptogenys | Lobopelta | rubicunda | Typen Borg. ] [ BRASIL, RIO DE JANEI- | RO, Petrópolis, IX | 1928, Wiltuschnij col. ] [ Coleção Entomológica Adoph | Hempel - Instituto Biológico | IBSP-IB-0.001.883 ].

01. [ 4744 ] [ Typus ] [ BRASIL, RIO DE JANEI- | RO, Petrópolis, IX | 1928, Wiltuschnij col. ] [ Coleção Entomológica Adoph | Hempel - Instituto Biológico | IBSP-IB-0.001.882 ].

01. [ 4744 ] [ Typus ] [ BRASIL, RIO DE JANEI- | RO, Petrópolis, IX | 1928, Wiltuschnij col. ] [ Coleção Entomológica Adoph | Hempel - Instituto Biológico | IBSP-IB-0.001.873 ].

02. [ 4744 || Leptogenys rubicunda | Ibid. (Petropolis. IX.28.Wiltuschnii.) ].

Megalomyrmex (Wheelerimyrmex) humilis Borgemeier, 1930

01. [ 3287 ] [Holotypus ] [ Megalomyrmex $\mid$ humilis Borgmeier $\mid=$ Pheidole $\mid$ gertrudae Forel $\mid$ n. syn. | W. W. Kempf. 1960 ] [ Megalomyrmex | (Weelerimyrmex) | humilis | Borg. ] [ Rio de Janeiro, RJ | 25.ii.1927 | 
O. Conde leg. ] [ BRASIL, RIO DE JANEI- | RO, Rio de Janeiro | 25.II.1927, Conde Col. ] [ Coleção Entomológica Adoph | Hempel - Instituto Biológico | IBSP-IB-0.002.188 ].

02. [ 3287 || Megalomyrmex pusilus var. | Ibid. (Rio. XII.27. Conde) ].

Megalomyrmex brasiliensis Borgmeier, 1930

01. [ 4425 ] [ Cotypus ] [ Megalomyrmex| (Weelerimyrmex) | gasparensis | brasiliensis | Borg. | Typen 4425 ] [Megalomyrmex | silvestrii | wh | Brandão det. ] [Gaspar SC | Manoel S. | Fontes leg. 1918] [Coleção Entomológica Adoph | Hempel - Instituto Biológico | IBSP-IB-0.003.198 ].

01. [4425 ] [Cotypus ] [ Gaspar SC | Manoel S. | Fontes leg. 1918] [ Megalomyrmex | silvestrii | wh | Brandão det. ] [ Coleção Entomológica Adoph | Hempel - Instituto Biológico | IBSP-IB-0.003.199 ].

01. [4425 ] [ Cotypus ] [ Gaspar SC | Manoel S. | Fontes leg. 1918] [ Megalomyrmex | silvestrii | wh | Brandão det. ] [ Coleção Entomológica Adoph | Hempel - Instituto Biológico | IBSP-IB-0.003.200 ].

02. [ 4425 || Megalomyrmex brasiliensis Borgm. | Ibid. (Gaspar. S. Cath. 1928, Fontes.) ].

Paracryptocerus fleddermanni Kempf, 1958

01. [ Coleção Entomológica Adoph | Hempel - Instituto Biológico | IBSP-IB-0.001.881] [ SÃO PAULO | ALTO DA SERRA | ARAUJO col. | II40 ] [ Paracryptocerus | fleddermanni | Kempf | PARATYPUS ].

Paracryptocerus frigidus Kempf, 1960

01. [ Coleção Entomológica Adoph | Hempel - Instituto Biológico | IBSP-IB-0.001.880 ] [ 1131 ] [ Cabo Frio RJ | VIII.1926 | Borgmeier | n 1131 ] [ Paracryptocerus | (H.) frigidus Kempf | Holotypus ] [ Paracryptocerus | frigidus Kempf | Kempf det. ].

02. [1131 || Cryptocerus frigidus | Ibid. (Cabo Frio. VIII.26 Borgm. ].

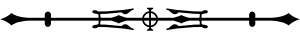

